KUCP-47

May, 1992

\title{
Differential Calculus on the Quantum Superspace and Deformation of Phase Space
}

\author{
Tatsuo Kobayashi周 and Tsuneo Uematsu f \\ Department of Physics, College of Liberal Arts and Sciences, \\ Kyoto University, Kyoto 606, Japan
}

\begin{abstract}
We investigate non-commutative differential calculus on the supersymmetric version of quantum space in which quatum supergroups are realized. Multiparametric quantum deformation of the general linear supergroup, $G L_{q}(m \mid n)$, is studied and the explicit form for the $\hat{R}$-matrix is presented. We apply these results to the quantum phase-space construction of $O S p_{q}(2 n \mid 2 m)$ and calculate their $\hat{R}$-matrices.
\end{abstract}

\footnotetext{
* Fellow of the Japan Society for the Promotion of Sience. Work partially supported by the Grantin-Aid for Scientific Research from the Ministry of Education, Science and Culture (\# 030083)

${ }^{\dagger}$ Work partially supported by the Grant-in-Aid for Scientific Research from the Ministry of Education, Science and Culture (\# 04245221)
} 


\section{Introduction}

Recently there has been much interest in quantum groups [1, 2, 3], in the context of studying integrable field theories, statistical models and conformal field theories in two dimensions (See, e.g. refs. [4, 烏).

Quantum groups can be realized on the quantum (hyper-)plane, in which coordinates are non-commuting [6, [7. Woronowicz has initiated the differential calculus on the non-commutative space of quantum groups [8], which provides an example for non-commutative differential geometry [9]. Wess and Zumino and others developed the differential calculus on the quantum (hyper-)plane covariant with respect to quantum groups [10] and multiparameter deformation of the quantum groups, especially for $G L_{q}(n)$ 11, 12, 13], as well as for $S O_{q}(n)$ [14].

Now it would be interesting to study q-deformation of supergroups from the viewpoint of quantum space. Quantum Lie superalgebras were studied in the framework of bosonic and fermionic q-oscillators [15, 16, 17]. The Manin's construction of quantum groups has already been extended to supergroups without recourse to differential calculus [18].

In this paper we investigate q-deformation of supersymmetric groups in the framework of differential calculus on the supersymmetric version of quantum hyper-plane or quantum superspace, where the non-commuting super-coordinates consist of bosonic as well as fermionic (Grassmann) coordinates. Based on the non-commutative differential calculus on the quantum superspace, we study quantum deformation of supergroups and $\hat{R}$-matrices which are solutions of the Yang-Baxter equation. We shall obtain the explicit form of the multiparametric $\hat{R}$-matrices for the quantum supergroup $G L_{q}(m \mid n)$. In contrast to the Manin's superspace [6, 18] we have the differential structure of the quantum superspace, therefore we can extend our results to quantum phase space of coordinates and momenta 110, 19, 20. Actually we shall apply our results for the $G L_{q}(m \mid n)$ to the phase space construction [19] of $O S p(2 n \mid 2 m)$-type quantum supergroups. [21, 22, 23].

This paper is organized as follows. In the next section we introduce the quantum superspace and study the differential calculus on it, in connection with the YangBaxter equation. In section 3 , the commutation relation of the matrix elements of quantum supergroups together with the quantum superdeterminant are investigated. 
Based on these results we construct the quantum phase-space of the $G L_{q}(m \mid n)$ in section 4 . The final section is devoted to some concluding remarks.

\section{Quantum superspace}

Now let us introduce the supersymmetric version of non-commuting coordinates which characterize the quantum supersymmetric hyper-plane or quantum superspace as follows:

$$
Z^{I}=\left(x^{i} ; \theta^{\alpha}\right) \quad(i=1, \cdots, m ; \alpha=1, \cdots, n)
$$

where $x^{i}$ and $\theta^{\alpha}$ denote bosonic and fermionic (Grassmannian) coordinates, respectively. They satisfy the following commutation relation

$$
Z^{I} Z^{J}=B_{K L}^{I J} Z^{K} Z^{L}
$$

We also set up the commutation relation between coordinates and differentials:

$$
Z^{I} d Z^{J}=C_{K L}^{I J} d Z^{K} Z^{L}
$$

We will see that these two coefficients $B^{I J}{ }_{K L}$ and $C_{K L}^{I J}$ are mutually related with each other from the consistency conditions. We now extend the differential calculus on quantum space developed by Wess and Zumino [10] and others [11-14] to this quantum superspace. Here we require that the exterior derivative $d$ given by

$$
d=d Z^{I} \frac{\partial}{\partial Z^{I}}=d x^{i} \frac{\partial}{\partial x^{i}}+d \theta^{\alpha} \frac{\partial}{\partial \theta^{\alpha}}
$$

obey the following two conditions:

i) nilpotency : $d^{2}=0$

ii) Leibniz rule: $d(f g)=(d f) g+(-1)^{\hat{f}} f(d g)$ where $\hat{f}$ denotes the Grassmann parity i.e. if $f$ is bosonic $\hat{f}=0$ and $\hat{f}=1$ if $f$ is a Grassmann variable.

From the Leibniz rule we have for an arbitrary function $f$

$$
d\left(Z^{I} f\right)=d Z^{I}\left(\frac{\partial}{\partial Z^{J}} Z^{I}\right) f+(-1)^{\hat{I}} Z^{I} d Z^{J} \frac{\partial}{\partial Z^{J}} f .
$$

Hence by using the commutation relation between the coordinates and the differentials (2.3) we get

$$
\frac{\partial}{\partial Z^{J}} Z^{I}=\delta_{J}^{I}+(-1)^{\hat{I}} C_{J L}^{I K} Z^{L} \frac{\partial}{\partial Z^{K}}
$$


We find the following equation holds

$$
\frac{\partial}{\partial Z^{R}} d Z^{K}=D_{R N}^{K M} d Z^{N} \frac{\partial}{\partial Z^{M}}
$$

where

$$
D_{R N}^{K M}=\left(C^{-1}\right)_{R N}^{K M} .
$$

This relation can be obtained by multiplying the both sides of (2.7) by $Z^{M}$ from the right and by making use of (2.6) together with the inverse of (2.3). Moreover, we multiply (2.3) by $\partial / \partial Z^{R}$ from the left and commute this derivative through to the right by using (2.6) and (2.7) we obtain

$$
(-1)^{\hat{I}} C_{R M}^{I M} D_{M T}^{J S} C_{U V}^{N T}=(-1)^{\hat{L}} C_{K L}^{I J} D_{R U}^{K M} C_{M V}^{L S}
$$

From the relation $(2.8)$ we get

$$
(-1)^{\hat{I}} C_{K L}^{I J} C_{S V}^{L N} C_{M Q}^{K S}=(-1)^{\hat{T}} C_{S U}^{J N} C_{M T}^{I S} C_{Q V}^{T U}
$$

Now we consider the relation between $B^{I J}{ }_{K L}$ and $C_{K L}^{I J}$. Acting a partial derivative $\partial / \partial Z^{M}$ on the commutation relation (2.2) from the left we extract the relation

$$
\left(\delta^{I}{ }_{K} \delta_{L}^{J}-B_{K L}^{I J}\right)\left(\delta_{M}^{K} \delta_{T}^{L}+(-1)^{\hat{K}} C_{M T}^{K L}\right)=0
$$

We further get a relation by taking the partial derivative of the relation (2.2) multiplied by an arbitrary function of $Z$ and using the commutaion relation (2.2) as

$$
(-1)^{\hat{I}+\hat{J}} C^{I P}{ }_{M T} C_{P U}^{J N} B_{Q V}^{T U}=(-1)^{\hat{K}+\hat{L}} B_{K L}^{I J} C_{M Q}^{K S} C_{S V}^{L N} .
$$

Now if we take

$$
B_{K L}^{I J}=(-1)^{\hat{I}} \frac{1}{X} C_{K L}^{I J},
$$

where $X$ is an arbitrary parameter, and substitute it into (2.12) we can recover the relation (2.10). Here we should note that the conservation of Grassmann parity : $\hat{I}+\hat{J}=\hat{K}+\hat{L}$ holds for the relation (2.2) as well as for the above equation. This can be proved from another derivation of the same relation by multiplying the differential of $Z$ on (2.2) from the right and commuting it through to the left. The parameter $X$ will turn out to be one of the deformation parameters. Thus we are led to the Yang-Baxter equation which is satisfied by $B_{K L}^{I J}$ given by (2.13)

$$
B_{K L}^{I J} B_{S V}^{L N} B_{M Q}^{K S}=B_{P U}^{J N} B_{M T}^{I P} B_{Q V}^{T U},
$$


which can be illustrated in Fig.1. This equation can be cast in the more familiar Yang-Baxter equation in terms of $\hat{R}$-matrices which are defined with the conventional normalization as follows

$$
B_{K L}^{I J}=\frac{1}{q} \hat{R}_{K L}^{I J} \quad, \quad q_{I J} \equiv q .
$$

In a similar argument which leads to eqs.(2.11) and (2.13), we can derive the following commutation relation:

$$
\partial_{I} \partial_{J}=B_{J I}^{L K} \partial_{K} \partial_{L} \quad, \quad \partial_{I} \equiv \partial / \partial Z^{I}
$$

Now let us restrict ourselves to the case of the q-deformed general linear supergroup $G L_{q}(m \mid n)$, for which we set up the following commutation relations

$$
\begin{aligned}
Z^{I} Z^{J} & =q_{I J} Z^{J} Z^{I} \\
d Z^{I} d Z^{J} & =-p_{I J} d Z^{J} d Z^{I} .
\end{aligned}
$$

Taking the exterior derivative of (2.3), generally we have

$$
d Z^{I} d Z^{J}=-(-1)^{\hat{K}} C_{K L}^{I J} d Z^{K} d Z^{L} .
$$

Noting that $\left(d x^{i}\right)^{2}=0,\left(\theta^{\alpha}\right)^{2}=0$ and using the relation (2.13) we explicit worked out the computation of $C^{I J}{ }_{K L}$. It turns out that $q_{I J}$ and $p_{I J}$ are related with each other by the equation

$$
p_{I J}=(-1)^{\hat{I}+\hat{J}} \frac{1}{X} q_{I J},
$$

and the explict form for the $B^{I J}{ }_{K L}$ or $\hat{R}$-matrix is found to be

$$
B^{I J}{ }_{K L}=\frac{1}{q} \hat{R}^{I J}{ }_{K L}=\delta^{I}{ }_{L} \delta^{J}{ }_{K}\left(\left(-\frac{1}{X}\right)^{\hat{I}} \delta^{I J}+\Theta^{I J} \frac{1}{q_{J I}}+\Theta^{J I} \frac{q_{I J}}{X}\right)+\delta^{I}{ }_{K} \delta^{J}{ }_{L} \Theta^{J I}\left(1-\frac{1}{X}\right)
$$

where $\Theta^{I J}$ is equal to 1 for $I>J$ and 0 for $I \leq J$, and $q$ denotes $q_{I J}$. This solution for the Yang-Baxter equation provides the multiparametric deformation of $G L(m \mid n)$ with $q_{I J}$ and $X$ as the deformation parameters. In the limit in which $q_{I J}$ and $X$ tend to 1 in (2.20), we can recover the classical case. Note that the whole $B$-matrix is composed of submatrices for a pair of the indices $I$ and $J(I<J)$ and is given by

$$
B_{(I, J)}=\left(\begin{array}{cccc}
\left(-\frac{1}{X}\right)^{\hat{I}} & 0 & 0 & 0 \\
0 & 1-\frac{1}{X} & \frac{q}{X} & 0 \\
0 & \frac{1}{q} & 0 & 0 \\
0 & 0 & 0 & \left(-\frac{1}{X}\right)^{\hat{J}}
\end{array}\right)
$$


and we find the eigenvalues for this submatrix are $1,-1 / X,(-1 / X)^{\hat{I}}$ and $(-1 / X)^{\hat{J}}$. Therefore, the eigenvalue equation of the whole $B$-matrix for $G L_{q}(m \mid n)(m+n=N)$ is given by

$$
\left\{(\lambda-1)\left(\lambda+\frac{1}{X}\right)\right\}^{N(N-1) / 2}(\lambda-1)^{m}\left(\lambda+\frac{1}{X}\right)^{n}=0 .
$$

Hence the characteristic equation satisfied by the $\hat{R}$-matrix is seen to be

$$
\left(\delta^{I}{ }_{K} \delta_{L}^{J}-B_{K L}^{I J}\right)\left(\delta_{M}^{K} \delta_{T}^{L}+X B_{M T}^{K L}\right)=0
$$

which is again consistent with (2.11) and (2.13). Note that for the case of $G L(2 \mid 0)$ $(G L(n \mid 0))$, (2.20) reduces to the well-known result for $\hat{R}$-matrix of the 2-parameter (the multiparameter) deformation of $G L(2)$ [11] $(G L(n)$ [12, 13]).(The multiparameter deformation of $G L(n)$ was also considered in ref. [24] on the basis of q-oscillators.) For $G L(2)$, the choice $: X=q^{2}$ leads to the well-known $\hat{R}$-matrix with one deformation parameter $q[10]$. One-parameter deformed $G L(1 \mid 1)$, also for the case $X=q^{2}$, was discussed in ref. 25]. By introducing projection operators $\mathcal{S}$ and $\mathcal{A}$ given as

$$
\mathcal{S}=\frac{1}{1+1 / X}\left(B+\frac{1}{X} \mathbf{1}\right) \quad, \quad \mathcal{A}=\frac{-1}{1+1 / X}(B-\mathbf{1})
$$

with the properties $\mathcal{S}^{2}=\mathcal{S}, \mathcal{A}^{2}=\mathcal{A}, \mathcal{S}+\mathcal{A}=\mathbf{1}$ and $\mathcal{A S}=\mathcal{S} \mathcal{A}=0$, the $B$-matrix can be expressed as

$$
B=\mathcal{S}-\frac{1}{X} \mathcal{A}
$$

Now we make some remarks on ordering of the indices. We distinguish two types of ordering between bosonic and fermionic(Grassmann) coordinates for $\hat{R}$-matrices of $G L_{q}(m \mid n)$, Type I $: i<\alpha$ and Type II $: i>\alpha$. Although this is not essential for the quantum-matrix commutation relation, these types lead to different expressions of the commutation relation between coordinates and partial derivatives given in (2.6).

Choosing the type I ordering, we can explicitly write down the expression of (2.6) for $G L_{q}(m \mid n)$ as follows

$$
\begin{aligned}
& \partial_{i} x^{i}=1+X x^{i} \partial_{i}+(X-1) \sum_{a=i+1}^{m} x^{a} \partial_{a}+(X-1) \sum_{\alpha=1}^{n} \theta^{\alpha} \partial_{\alpha} \\
& \partial_{J} Z^{I}=q_{I J} Z^{I} \partial_{J} \quad, \quad \partial_{I} Z^{J}=\frac{X}{q_{I J}} Z^{J} \partial_{I} \quad, \quad I<J \\
& \partial_{\alpha} \theta^{\alpha}=1-\theta^{\alpha} \partial_{\alpha}-(1-X) \sum_{\beta=\alpha+1}^{n} \theta^{\beta} \partial_{\beta} .
\end{aligned}
$$


A similar but different expression can also be obtained for the type II ordering.

\section{Quantum group and superdeterminant}

The coordinates of the superspace, $Z^{J}$ are linearly transformed by a matrix $T_{J}^{I}$ into new coordinates $Z^{I I}$, i.e., $Z^{I I}=T_{J}^{I} Z^{J}$. Covariance of (2.2) requires commutation relations of $T_{J}^{I}$,

$$
(-1)^{\hat{K}(\hat{N}+\hat{L})} B_{M N}^{I J} T_{K}^{M} T_{L}^{N}=(-1)^{\hat{M}(\hat{J}+\hat{N})} T_{M}^{I} T_{N}^{J} B_{K L}^{M N},
$$

which was also discussed in ref.[18].

Using (2.20), we obtain explicit commutaion relations of $T_{J}^{I}$,

$$
\begin{gathered}
T_{K}^{I} T_{K}^{J}=(-1)^{\hat{K}(\hat{I}+\hat{J})}\left(-\frac{1}{X}\right)^{\hat{K}} q_{I J} T_{K}^{J} T_{K}^{I}, \\
T_{K}^{I} T^{I}=(-1)^{\hat{I}(\hat{K}+\hat{L})}\left(-\frac{1}{X}\right)^{\hat{I}} \frac{X}{q_{K L}} T_{L}^{I} T_{K}^{I}, \\
T_{L}^{I} T_{K}^{J}=(-1)^{\hat{K} \hat{I}+\hat{J} \hat{L}} \frac{q_{I J} q_{K L}}{X} T_{K}^{J} T_{L}^{I}, \\
T_{K}^{I} T_{L}^{J}=(-1)^{\hat{K} \hat{J}+\hat{I} \hat{L}} \frac{q_{I J}}{q_{K L}} T^{J}{ }_{L} T_{K}^{I}+(-1)^{\hat{J}(\hat{K}+\hat{L})} \frac{X-1}{q_{K L}} T_{L}^{I} T_{K}^{J},
\end{gathered}
$$

where $I<J$ and $K<L$. The relations show that $T_{J}^{I}$ are nothing but matrix elements of $G L_{q}(m \mid n)$ quantum group, which has most deformation parameters we have ever known. It is remarkable that transposed matrix elements satisfy the same relations except $q_{I J}$ replacing $X / q_{I J}$.

Next we define a superdeterminant of the quantum matrix $T$. The superdeterminant for $G L_{q}(1 \mid 1)$ has been obtained in refs. $[17,25]$. Here we denote $T$ by block matrices,

$$
T_{J}^{I}=\left(\begin{array}{cc}
A_{j}^{i} & B_{\beta}^{i} \\
C_{j}^{\alpha} & D_{\beta}^{\alpha}
\end{array}\right),
$$

where $A(D)$ transforms bosonic (fermionic) coordinates into new bosonic (fermionic) coordinates. At first we consider the case where only fermionic coordinates are linearly transformed one another. The fermionic determinant of the matrix $D$ is defined through an equation;

$$
\prod_{\alpha=1}^{n} \theta^{\alpha}=\operatorname{det}_{f} D \prod_{\alpha=1}^{n} \theta^{\alpha}
$$


If we introduce a fermionic volume element $\mathcal{D} \theta$ as

$$
\int\left(\prod_{\alpha=1}^{n} \theta^{\alpha}\right) \mathcal{D} \theta=1
$$

we find

$$
\mathcal{D} \theta^{\prime}=\left(\operatorname{det}_{f} D\right)^{-1} \mathcal{D} \theta
$$

On the other hand, in transformation of only bosonic coordinates, the bosonic determinant of the matrix $A$ is defined as ref.[11,12] through an equation,

$$
\prod_{k=1}^{m} d x^{\prime k}=\operatorname{det}_{b} A \prod_{k=1}^{m} d x^{k} .
$$

Then superdeterminant of the matrix $T$ is defined using the above volume elements,

$$
\left(\prod_{k=1}^{m} d x^{\prime k}\right) \mathcal{D} \theta^{\prime}=\operatorname{Sdet} T\left(\prod_{k=1}^{m} d x^{k}\right) \mathcal{D} \theta .
$$

Note that the matrix $T$ can be decomposeed into a product of three simple matrices;

$$
\left(\begin{array}{cc}
A & B \\
C & D
\end{array}\right)=\left(\begin{array}{cc}
1 & B D^{-1} \\
0 & 1
\end{array}\right)\left(\begin{array}{cc}
\tilde{A} & 0 \\
0 & D
\end{array}\right)\left(\begin{array}{cc}
1 & 0 \\
D^{-1} C & 1
\end{array}\right),
$$

where $\tilde{A}=A-B D^{-1} C$. Only the second matrix of the right hand side contributes to the superdeterminant, because the volume element is not changed under the translation. Thus we find $\operatorname{Sdet} T=\operatorname{det}_{b} \tilde{A}\left(\operatorname{det}_{f} D\right)^{-1}$.

Using (2.20), we obtain explicitly the bosonic and fermionic determinants as

$$
\begin{aligned}
& \operatorname{det}_{b} A=\sum_{\sigma}\left(\prod_{i<k, \sigma(i)>\sigma(k)}\left(-p_{\sigma(i) \sigma(k)}\right)^{-1}\right) A_{\sigma(1)}^{1} A_{\sigma(2)}^{2} \cdots A_{\sigma(m)}^{m}, \\
& \operatorname{det}_{f} D=\sum_{\sigma}\left(\prod_{\alpha<\beta, \sigma(\alpha)>\sigma(\beta)}\left(q_{\sigma(\alpha) \sigma(\beta)}\right)^{-1}\right) D_{\sigma(1)}^{1} D_{\sigma(2)}^{2} \cdots D_{\sigma(n)}^{n},
\end{aligned}
$$

where $\sigma$ implies a possible permutation. When $q_{I J}=X=1$, the bosonic and the fermionic determinants and the superdeterminant become "classical" obviously.

Further,we obtain a commutaton relation,

$$
x^{i} \prod_{k=1}^{m} d x^{k}=\left(\prod_{k=1}^{m} d x^{k}\right) x^{i} c_{(i)} X^{i}, \quad d \theta^{\alpha} \prod_{\beta=1}^{n} \theta^{\beta}=\left(\prod_{\beta=1}^{n} \theta^{\beta}\right) d \theta^{\alpha} c_{(\alpha)} X^{\alpha-n},
$$

where

$$
c_{(i)} \equiv\left(\prod_{k=1}^{i-1} \frac{1}{q_{k i}}\right) \prod_{k=i+1}^{m} q_{i k}, \quad c_{(\alpha)} \equiv\left(\prod_{\beta=1}^{\alpha-1} \frac{1}{q_{\beta \alpha}}\right) \prod_{\beta=\alpha+1}^{n} q_{\alpha \beta} .
$$


This leads to commutation relations between the bosonic determinant and each matrix element $T_{j}^{i}$,

$$
c_{(j)} X^{j} T_{j}^{i} \operatorname{det}_{b} \tilde{A}=c_{(i)} X^{i} \operatorname{det}_{b} \tilde{A} T_{j}^{i},
$$

which coincides with the result of ref.[12]. In addition to (3.11), we use the commutation relations of the transposed matrix to find commutaion relations between the superdeterminant and the matrix elements $T_{J}^{I}$. Thus, we obtain relations,

$$
\begin{gathered}
T_{j}^{i} \mathcal{T}=X^{i-j} \frac{c_{(i)}}{c_{(j)}}\left(\prod_{\beta=1}^{n} \frac{q_{j \beta}}{q_{i \beta}}\right) \mathcal{T} T^{i}{ }_{j}, \quad T_{\beta}^{\alpha} \mathcal{T}=X^{\beta-\alpha} \frac{c_{(\beta)}}{c_{(\alpha)}}\left(\prod_{k=1}^{m} \frac{q_{k \beta}}{q_{k \alpha}}\right) \mathcal{T} T_{\beta}^{\alpha}, \\
T^{i}{ }_{\alpha} \mathcal{T}=X^{i+\alpha-m-1} c_{(i)} c_{(\alpha)} \frac{\prod_{k=1}^{m} q_{k \alpha}}{\prod_{\beta=1}^{n} q_{i \beta}} \mathcal{T} T^{i}{ }_{\alpha},
\end{gathered}
$$

where $\mathcal{T} \equiv \operatorname{Sdet} T$. We can find commutation relations between the superdeterminant and $T_{i}^{\alpha}$, using the fact that the superdeterminant and $T_{J}^{I} T_{I}^{J}$ commute each other.

The centrality of the superdeterminant requires

$$
\frac{X^{i} c_{(i)}}{\prod_{\beta=1}^{n} q_{i \beta}}=\frac{X^{-\alpha-m-1} c_{(\alpha)}^{-1}}{\prod_{k=1}^{m} q_{k \alpha}}=\mathrm{const},
$$

for all $i$ and $\alpha$. In general, eq. (3.15) provides $(m+n-1)$ independent conditions on the deformation parameters, while in special case where $m=n$ we find only $(m+n-2)$ independent conditions. Therefore, we can obtain $S L_{q}(m \mid n)$ quantum groups with $\{(N-2)(N-1) / 2+1\}$ independent deformation parameters and $S L_{q}(m \mid m)$ quantum groups with $\{(m-1)(2 m-1)+2\}$ independent parameters. The latter differs from the situation of $S L_{q}(m)$ of ref.[12] and that is one of remarkable aspects of the quantum supergroups.

\section{Deformed phase space}

In this section, we deform phase space of the supersymmetric coordinates and momenta, following refs.[10,19]. We consider the real quantum superspace, i.e., $\bar{Z}^{I}=$ $Z^{I}$. That requires conditions on the deformation parameters, i.e., $\bar{q}_{I J}=1 / q_{I J}, \bar{X}=$ $1 / X$. Further, the derivatives should satisfy

$$
\bar{\partial}_{i}=-X^{2 \rho(i)} \partial_{i}, \quad \bar{\partial}_{\alpha}=X^{2 \rho(\alpha)} \partial_{\alpha},
$$


where $\rho(i)=(m+1-n-i) / 2, \rho(\alpha)=(\alpha-n) / 2$ in the case of Type I ordering and $\rho(i)=(m+1-i) / 2, \rho(\alpha)=(m+\alpha-n) / 2$ for Type II ordering. Therefore we can define real momentum operators as

$$
p_{i} \equiv-i X^{\rho(i)} \partial_{i}, \quad p_{\alpha} \equiv X^{\rho(\alpha)} \partial_{\alpha}
$$

Eqs.(2.2), (2.6) and (2.16) requires that these super phase-space should follow commutation relations,

$$
\begin{gathered}
Z^{I} Z^{J}=B_{K L}^{I J} Z^{K} Z^{L}, \quad P_{L} P_{K}=B_{K L}^{I J} P_{J} P_{I}, \\
P_{K} Z^{I}=-(i)^{\hat{K}+1} C_{K}^{I}+(i)^{\hat{K}-\hat{J}} X^{\rho(K)-\rho(J)+1} B_{K L}^{I J} Z^{L} P_{J},
\end{gathered}
$$

where $C^{I}{ }_{K}=X^{\rho(K)} \delta_{K}^{I}$ and $P_{I}=\left(p_{i}, p_{\alpha}\right)$.

Suppose that we define super-gamma matrices as

$$
\gamma^{\alpha}=p_{\alpha}, \quad \gamma^{2 n-\alpha+1}=\theta^{\alpha}, \quad c^{i}=p_{i}, \quad c^{2 m-i+1}=x^{i} .
$$

Hereafter we denote $\alpha^{\prime}=2 n-\alpha+1$ and $i^{\prime}=2 m-i+1$. We can derive deformed super-Heisenberg algebra or "super"-Clifford algebra from the phase space algebra as follows,

$$
\Gamma^{I} \Gamma^{J}+\left(-\tilde{B}_{K L}^{I J}\right) \Gamma^{K} \Gamma^{L}=C^{I J}(-i)^{\hat{I}+1}
$$

where $\Gamma^{I}=\left(\gamma^{\alpha}, c^{i}\right)(\alpha=1, \cdots, 2 n, i=1, \cdots, 2 m)$. The matrix $\tilde{B}_{K L}^{I J}$ is ,of course, related to the matrix $B_{K L}^{I J}$, i.e.,

$$
\begin{gathered}
\tilde{B}_{K^{\prime} L^{\prime}}^{I^{\prime}}=B_{K L}^{I J}, \quad \tilde{B}_{K L}^{I J}=B_{J I}^{L K}, \\
\tilde{B}_{K^{\prime} L}^{I J^{\prime}}=(i)^{\hat{I}-\hat{L}} X^{\rho(I)-\rho(L)+1} B_{I K}^{J L}, \\
\tilde{B}_{K L^{\prime}}^{I^{\prime}{ }_{I K}}=(i)^{\hat{J}-\hat{K}} X^{\rho(J)-\rho(K)-1}\left(B^{-1}\right)_{J L}^{I K},
\end{gathered}
$$

where $I, J, K, L<m+1(n+1)$ in the bosonic (fermionic) case and the inverse of the matrix $B$ is defined as $\left(B^{-1}\right)_{J K}^{L I} B_{K N}^{I M}=\delta^{L M} \delta_{J N}$. Further the metric matrix $C$ is obtained as $C^{I J^{\prime}}=C_{I}^{J}, C^{I J}=0$ and so on. Among the block matrices of the matrix $\tilde{B}, \tilde{B}_{K^{\prime} L^{\prime}}^{I^{\prime}}$ and $\tilde{B}_{K L}^{I J}$ of (4.6.a) have eigenvalues 1 and $-1 / X$, as said in section two. The other block matrix with (4.6.b) and (4.6.c) as off-diagonal sub-matrices, has eigenvalues \pm 1 . It is easily shown that the matrix $\tilde{B}$ satisly the Yang-Baxter equation. Suppose that we graphically represent the $\tilde{B}_{K^{\prime} L}^{I J^{\prime}}$ of (4.6.b) as Fig.2. It is related to the diagram of $B_{I K}^{J L}$. For example one set of the Yang-Baxter equations 
with indices shown in Fig.3 is identified with the equation represented by Fig.1.b, up to an overall factor, $(i)^{\hat{M}-\hat{N}} X^{\rho(M)-\rho(N)+1}$. Similarly, we can prove that the matrix $\tilde{B}$ with the other indices satisfy the Yang-Baxter equation, making use of the fact that $B$ is the solution of the Yang-Baxter equation and one sort of conservation law that the matrix elements $B_{K L}^{I J}$ vanish unless $I+J=K+L$ and $I-J=K-L$. Imposing conditions, $q_{I J}=q$ and $X=q^{2}$, we find that the $\tilde{B}$ matrices for the $G L_{q}(m \mid 0)$ and $G L_{q}(0 \mid n)$ phase spaces coincide with well-known $\hat{R}$-matrices of $S p_{q}(2 m)$ and $S O_{q}(2 n)$ deformed by one parameter, respectively, as shown in ref.[19]. Further, the above analysis suggests $\hat{R}$-matrices for deformed $O S p(2 n \mid 2 m)$ groups.

Moreover, we can define a quantum superspace with a metric, whose differentials satisfy commutation relations,

$$
d \tilde{Z}^{I} d \tilde{Z}^{J}=\tilde{B}_{K L}^{I J} d \tilde{Z}^{K} d \tilde{Z}^{L}
$$

where new coordinates $\tilde{Z}^{I}$ in a deformed superspace consist of new bosonic coordinates $\tilde{x}^{\alpha}(\alpha=1 \cdots 2 n)$ and new fermionic coordinates $\tilde{\theta}^{i}(i=1 \cdots 2 m)$, i.e., grassmann parities of all new coordinates are reversed, compared with the orginal $G L_{q}(m \mid n)$ phase space. From (4.7), we can derive commutation relations between the new coordinates and the differentials by the discussion on (2.3) and (2.18) as

$$
\tilde{Z}^{I} d \tilde{Z}^{J}=-(-1)^{\hat{K}} \tilde{B}_{K L}^{I J} d \tilde{Z}^{K} \tilde{Z}^{L}
$$

Further, using characteristic equations of the block matrices of $\tilde{B}$ and (2.11), we can derive commutaions relations for the new coordinates $\tilde{Z}$ in a similar way to the procedure in the section two.

Let us consider the $G L_{q}(1 \mid 1)$ phase space as a concrete example, from which we derive $\hat{R}$-matrix for deformed $O S p(2 \mid 2)$. The deformation of $O S p(2 \mid 2)$ was also discussed algebraically in ref.[23]. In the case of Type I ordering, bosonic and fermionic momenta are defined as,

$$
p_{x} \equiv-i \partial_{x}, \quad p_{\theta} \equiv \partial_{\theta}
$$

They and the coordinates satisfy commutaion relations as follows,

$$
\begin{gathered}
p_{x} p_{\theta}=\frac{q}{X} p_{\theta} p_{x}, \quad x \theta=q \theta X, \\
p_{x} \theta=\frac{X}{q} \theta p_{x}, \quad p_{\theta} x=q x p_{\theta}, \\
p_{x} x=-i+X x p_{x}-i(X-1) \theta p_{\theta},
\end{gathered}
$$




$$
p_{\theta} \theta=1-\theta p_{\theta}, \quad p_{\theta}^{2}=\theta^{2}=0,
$$

where these relations are dominated by the matrix $\tilde{B}$ constructed as (4.6). In this case, the matrix $\tilde{B}$ is obtained explicitly as

$$
\begin{gathered}
\tilde{B}=\left(\begin{array}{cccc}
a^{*} & & & \\
& 0 & b & \\
& b^{-1} & 0 & \\
& & & a
\end{array}\right), \\
a=\left(\begin{array}{cccc}
-1 / X & & & \\
& 0 & 1 / q & \\
& 1 / X & 1-1 / X & \\
& & 1
\end{array}\right), b=\left(\begin{array}{cccc}
-1 & & & \\
& & & \\
& X / q & 0 & \\
i(1-X) & & & X
\end{array}\right),
\end{gathered}
$$

where $a^{*}$ is obtained from $a$ by rearrangement of the elements as (4.6.a). Suppose that we define gamma matrices $\gamma^{1} \equiv p_{\theta}, \gamma^{2} \equiv \theta, c^{1} \equiv p_{x}$ and $c^{2} \equiv x$. They satisfy super-Clifford algebra for deformed $O S p(2 \mid 2)$ group as

$$
\begin{array}{cc}
\left(\gamma^{1}\right)^{2}=\left(\gamma^{2}\right)^{2}=0, & \gamma^{1} \gamma^{2}+\gamma^{2} \gamma^{1}=1, \\
c^{1} c^{2}-X c^{2} c^{1}+i(X-1) \gamma^{2} \gamma^{1}=-i, \\
c^{1} \gamma^{1}-\frac{q}{X} \gamma^{1} c^{1}=0, & \gamma^{1} c^{2}-q c^{2} \gamma^{1}=0, \\
c^{1} \gamma^{2}-\frac{X}{q} \gamma^{2} c^{1}=0, & c^{2} \gamma^{2}-q \gamma^{2} c^{2}=0 .
\end{array}
$$

Using the matrix $\tilde{B}$ as (4.7), we can define commutation relations between differentials of the deformed $O S p(2 \mid 2)$ superspace as,

$$
\begin{gathered}
\left(d \tilde{x}^{1}\right)^{2}=\left(d \tilde{x}^{2}\right)^{2}=0, \quad d \tilde{x}^{1} d \tilde{x}^{2}=-d \tilde{x}^{2} d \tilde{x}^{1}, \\
d \tilde{\theta}^{1} d \tilde{\theta}^{2}=X d \tilde{\theta}^{2} d \tilde{\theta}^{1}-i(X-1) d \tilde{x}^{2} d \tilde{x}^{1} \\
d \tilde{\theta}^{1} d \tilde{x}^{1}=\frac{q}{X} d \tilde{x}^{1} d \tilde{\theta}^{1}, \quad d \tilde{\theta}^{1} d \tilde{x}^{2}=\frac{X}{q} d \tilde{x}^{2} d \tilde{\theta}^{1}, \\
d \tilde{x}^{1} d \tilde{\theta}^{2}=q d \tilde{\theta}^{2} d \tilde{x}^{1}, \quad d \tilde{x}^{2} d \tilde{\theta}^{2}=q d \tilde{\theta}^{2} d \tilde{x}^{2} .
\end{gathered}
$$

Here we define another parity $\epsilon(I)$ as $\epsilon(1)=0$ and $\epsilon(2)=1$ in both bosonic and fermionic cases. The matrix $\tilde{B}_{K L}^{I J}$ satisfy the characteristic equation,

$$
\left(\tilde{B}_{K L}^{I J}-\delta_{K}^{I}{ }_{K}^{J}{ }_{K}\right)\left(\tilde{B}_{K L}^{I J}+\left(\frac{1}{X}\right)^{|\epsilon(I)-\epsilon(J)|} \delta_{K}^{I}{ }_{K}^{J}{ }_{L}\right)=0
$$


and also $(-1)^{\hat{I}+\hat{K}} \tilde{B}_{K L}^{I J}$ satisfy the same equation as (4.14), where $\hat{I}$ and $\hat{K}$ denote the grassmann parities of the new coordinates. Using the characteristic equation (4.14), we can easily decompose the matrix $\tilde{B}_{K L}^{I J}$ into projection operators, as said in section two. Then we will derive a matrix $\tilde{B}_{K L}^{\prime I J}$ which dominates commutation relations between new coordinates $\tilde{Z}^{I}$ as $\tilde{Z}^{I} \tilde{Z}^{J}=\tilde{B}_{K L}^{\prime I J} \tilde{Z}^{K} \tilde{Z}^{L}$. Comparing the characteristic equation (4.14) with (2.11), we find

$$
\tilde{B}_{K L}^{\prime I J}=-(-1)^{\hat{I}+\hat{K}} X^{|\epsilon(I)-\epsilon(J)|} \tilde{B}_{K L}^{I J},
$$

and explicitly obtain the commutation relations as

$$
\begin{array}{cc}
\tilde{x}^{1} \tilde{x}^{2}=\tilde{x}^{2} \tilde{x}^{1}, & \left(\tilde{\theta}^{1}\right)^{2}=\left(\tilde{\theta}^{2}\right)^{2}=0, \\
\tilde{\theta}^{1} \tilde{x}^{1}=q \tilde{x}^{1} \tilde{\theta}^{1}, & \tilde{x}^{1} \tilde{\theta}^{2}=q \tilde{\theta}^{2} \tilde{x}^{1}, \\
\tilde{\theta}^{1} \tilde{x}^{2}=\frac{X}{q} \tilde{x}^{2} \tilde{\theta}^{1}, & \tilde{x}^{2} \tilde{\theta}^{2}=\frac{X}{q} \tilde{\theta}^{2} \tilde{x}^{2}, \\
\tilde{\theta}^{1} \tilde{\theta}^{2}=-X \tilde{\theta}^{2} \tilde{\theta}^{1}-i(X-1) \tilde{x}^{1} \tilde{x}^{2} .
\end{array}
$$

This algebra has a center, $\tilde{x}^{1} \tilde{x}^{2}+i \tilde{\theta}^{1} \tilde{\theta}^{2}$, i.e., it is not deformed from the classical one. It can be described as

$$
\tilde{x}^{i} \tilde{C}_{i j} \tilde{x}^{j}+i \tilde{\theta}^{\alpha} \tilde{C}_{\alpha \beta}^{\prime} \tilde{\theta}^{\beta} \equiv \frac{X+1}{4}\left(\tilde{x}^{1} \tilde{x}^{2}+\tilde{x}^{2} \tilde{x}^{1}\right)+i \frac{\sqrt{X}}{2}\left(\frac{1}{\sqrt{X}} \tilde{\theta}^{1} \tilde{\theta}^{2}-\sqrt{X} \tilde{\theta}^{2} \tilde{\theta}^{1}\right) .
$$

It is remarkable that ratios of the metrics, $\tilde{C}_{12} / \tilde{C}_{21}$ and $\tilde{C}_{12}^{\prime} / \tilde{C}_{21}^{\prime}$, coincide with those of $S O_{q}(2)$ and $S p_{q}(2)$ quantum groups of ref.[7], respectively. By similar procedure, we can derive commutaion relations of the deformed $O S p(2 \mid 2)$ superspace for Type II ordering, where we can find that $\tilde{x}^{1} \tilde{x}^{2}+i \tilde{\theta}^{1} \tilde{\theta}^{2}$ is also central. Further the similar analysis on the $G L_{q}(m \mid n)$ phase space lead to various quantum superspaces with metrics.

\section{Concluding remarks}

In this paper we investigated the differential calculus on the non-commutative superspace. We obtained the Yang-Baxter equation in this framework for quantum supergroups, and in particular, the explicit form for the multiparametric $\hat{R}$-matrix in the case of q-deformed general linear supergroup, $G L_{q}(m \mid n)$. We also presented the commutation relation of quantum supergroup matrix-elements as well as quantum 
superdeterminants. We then extended these results to phase-space construction of $O S p_{q}(2 n \mid 2 m)$. Some details for quantum super-Clifford algebras and the $\hat{R}$-matrix were presented for the case of $O S p_{q}(2 \mid 2)$.

It would be interesting to extend the present result to more general supergroups. It should also be worked out to find the relation between the quantum group and the quantum Lie algebras. In the context of differential calculus, one of the next issues to be studied is the quantum differential form [26].

Finally, there have been a number of works on quantum deformations of spacetime symmetries such as Lorentz [27, 28, 29] and Poincaré [30, 31, 32] groups in the literatures. In this connection, the most intriguing subject to be pursued further might be q-deformation of super-Poincaré symmetry.

The authors would like to thank T. Inami, P. Kulish and R. Sasaki for valuable discussions. 


\section{References}

[1] V. G. Drinfeld, Sov. Math. Dokl. 32(1985)254.

[2] M. Jimbo, Lett. Math. Phys. 10(1985)63.

[3] L. D. Faddeev, N. Yu. Reshetikhin and L. A. Takhtajan, Algebra and Analysis, $\mathbf{1}(1987) 178$.

[4] H. -D. Doebner and J. -D. Hennig eds., Quantum Groups, Lecture Notes in Physics 370(Springer Verlag, 1990).

[5] T. Curtright, D. Fairlie and C. Zachos eds., Quantum Groups, Proceedings of the Argonne Workshop (World Scientific, 1990).

[6] Yu. I. Manin, Commun. Math. Phys. 123(1989)163.

[7] L. A. Takhtajan, Adv. Studies Pure Math. 19(1989)435; Lecture Notes in Physics 370(1989)3.

[8] S. L. Woronowicz, Commun. Math. Phys. 111(1987)613; 122(1989)125.

Publ. RIMS, Kyoto Univ. 23(1987)117.

[9] A. Connes, Publ. Math. IHES 62(1985)41.

[10] J. Wess and B. Zumino, Nucl. Phys. B (Proc. Suppl.) 18B(1990)302.

[11] A. Schirrmacher, J. Wess and B. Zumino, Z. Phys. C 49(1991)317.

[12] A. Schirrmacher, Z. Phys. C 50(1991)321.

[13] A. Schirrmacher, J. Phys. A: Math. Gen. 24(1991)L1249.

[14] U. Carow-Watamura, M. Schlieker and S. Watamura, Z. Phys. C 49(1991) 439.

[15] P. P. Kulish, "Quantum Groups and Quantum Algebras as Symmetries of Dynamical Systems ", Preprint YITP/K-959 (1991);

Phys. Lett. A161(1991)50.

[16] M. Chaichan and P. Kulish, Phys. Lett. 234B(1990)72.

[17] M. Chaichan, P. Kulish and J. Lukierski, Phys. Lett. 237B(1990)401; Phys. Lett. 262B(1991)43. 
[18] E. Corrigan, D. B. Fairlie, P. Fletcher and R. Sasaki, J. Math.Phys. 31(1990)776.

[19] B. Zumino, Mod. Phys. Lett. A6(1991)1225.

[20] I. Ya. Aref'eva and I. V. Volovich, Phys. Lett. B268(1991)179.

[21] P. P. Kulish, "Quantum Superalgebra OSp(1|2)", Preprint RIMS-615 (1988);

P. P. Kulish and N. Yu. Reshetikhin, Lett. Math. Phys. 18(1989)143;

E. Celeghini, R. Giachetti, P. P. Kulish, E. Sorace and M. Tarlini, J. Phys. A:Math. Gen. 24(1991)5675.

[22] H. Saleur, Nucl. Phys. B336(1990)363.

[23] T. Deguchi, A. Fujii and K. Ito, Phys, Lett. 238B(1990)242.

[24] D. B. Fairlie and C. K. Zachos, Phys. Lett. 256B(1991)43.

[25] W. B. Schmidke, S. P. Vokos and B. Zumino, Z. Phys. C 48(1990)249.

[26] B. Zumino, "Introduction to the Differential Geometry of Quantum Groups ", Preprint LBL-31432;UCB-PTH-62/91 (1991).

[27] U. Carow-Watamura, M. Schlieker, M. Scholl and S. Watamura, Z. Phys. C 48(1990)159; Int. J. Mod. Phys. A6(1991)3081.

[28] P. Podleś and S. L. Woronowicz, Commun. Math. Phys. 130(1990)381.

[29] W. B. Schmidke, J. Wess and B. Zumino, Z. Phys. C 52(1991)471.

[30] J. Lukierski, H. Ruegg, A. Nowicki and V. N. Tolstoy, Phys. Lett. 264B (1991)331.

[31] J. Lukierski, A. Nowicki and H. Ruegg, "Quantum Deformation of Poincaré and Conformal Algebras", Preprint BUHEP-91-21(1991).

[32] O. Ogievetsky, W. B. Schmidke, J. Wess and B. Zumino, "q-Deformed Poincaré Algebra ", Preprint MPI-Ph/91-98; LBL-31703;UCB 92/04 (1991). 
$\mathrm{B}_{\mathrm{KL}}^{\mathrm{IJ}}={ }_{\mathrm{K}}^{\mathrm{I}} \overbrace{\mathrm{L}}^{\mathrm{J}}$

a

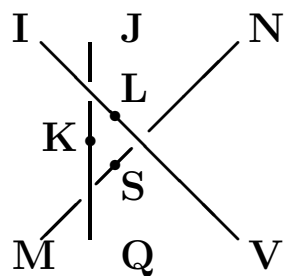

b

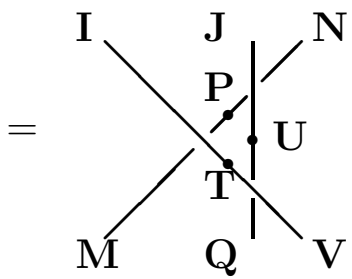

b

Fig.1.a,b Graphical representations of the matrix $B$ and the Yang-Baxter equation

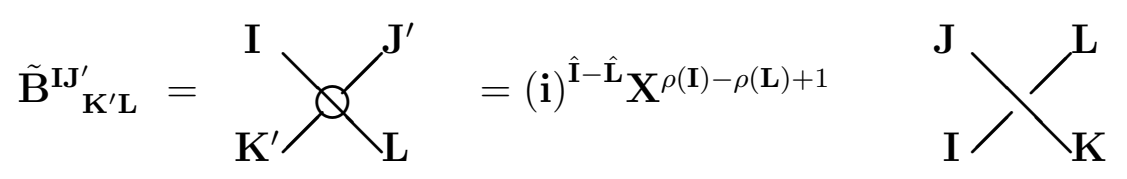

Fig.2 Graphical representation of the block matrix of $\tilde{\mathbf{B}}$ written as eq(4.6.b)

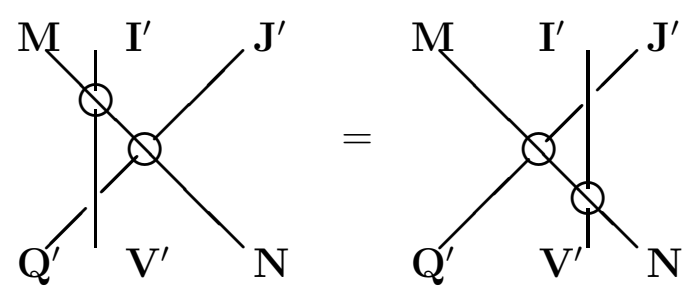

Fig.3 Graphical representation of some of the Yang-Baxter equations of $\tilde{B}$ 